\title{
Generalized Collision-free Velocity Model for Pedestrian Dynamics
}

\author{
Qiancheng $\mathrm{Xu}^{\mathrm{a}, *}$, Mohcine Chraibi $^{\mathrm{a}}$, Antoine Tordeux ${ }^{\mathrm{b}}$, Jun Zhang ${ }^{\mathrm{c}}$ \\ ${ }^{a}$ Institute for Advanced Simulation, \\ Forschungszentrum Jülich GmbH, 52425 Jülich, Germany \\ ${ }^{b}$ School of Mechanical Engineering and Safety Engineering, \\ University of Wuppertal, 42119 Wuppertal, Germany \\ ${ }^{c}$ State Key Laboratory of Fire Science, \\ University of Science and Technology of China, 230027 Hefei, China
}

\begin{abstract}
The collision-free velocity model is a microscopic pedestrian model, which despite its simplicity, reproduces fairly well several self-organization phenomena in pedestrian dynamics. The model consists of two components: a direction sub-model that combines individual desired moving direction and neighbor's influence to imitate the process of navigating in a two-dimensional space, and an intrinsically collision-free speed sub-model which controls the speed of the agents with respect to the distance to their neighbors.

In this paper we generalize the collision-free velocity model by introducing the influence of walls and extending the distance calculations to velocity-based ellipses. Besides, we introduce enhancements to the direction sub-module that smooth the direction changes of pedestrians in the simulation; a shortcoming that was not visible in the original model due to the symmetry of the circular shapes. Moreover, the introduced improvements mitigate backward movements, leading to a more realistic distribution of pedestrians especially in bottleneck scenarios.

We study by simulation the effects of the pedestrian's shape by comparing the fundamental diagram in narrow and wide corridors. Furthermore, we validate our generalized approach by investigating the flow through bottlenecks with varying exit's widths.
\end{abstract}

Keywords: Collision-free velocity model, pedestrian dynamics, dynamical ellipse, fundamental diagram, validation

\section{Introduction}

Nowadays, the scale of crowd activities is getting bigger with the constant increase in the world population and the convenience of transport. Although these events usually are

\footnotetext{
*I am corresponding author

Email addresses: q.xu@fz-juelich.de (Qiancheng Xu), m.chraibi@fz-juelich.de (Mohcine Chraibi), tordeux@uni-wuppertal.de (Antoine Tordeux), junz@ustc.edu.cn (Jun Zhang)
} 
carefully planned before they are held, the probability of accidents cannot be neglected, especially when the number of participants is considerably high. Besides, in some complex buildings, such as train stations, airports, stadiums, and commercial malls, crowd density can be relatively high, in particular during rush hours. For increasing the comfort and usability of these facilities, simulations of pedestrian dynamics may help during the design of buildings and even after their construction to identify potential bottlenecks and mitigate their effects $[1,2]$.

In general, models used to describe pedestrian dynamics can be categorized according to their scale of definition into macroscopic models, mesoscopic models, and microscopic models. Microscopic models describe individual trajectories of pedestrians while macroscopic models rely on aggregated quantities e.g. density, velocity, and flow to describe pedestrian dynamics in partial differential equation systems $[3,4,5,6]$. For instance, recent macroscopic approaches rely on mean-field game theory and the coupling of Hamilton-Jacobi-Bellman and Fokker-Planck equations $[7,8,9,10]$. The intermediate scale between microscopic and macroscopic classes is mesoscopic. Kinetic models [11, 12, 13, 14, 15] describing the crowd through distribution functions with Boltzmann-type equations and discrete queuing models $[16,17,18]$ belong to the mesoscopic modeling category. Mesoscopic models can take in consideration behavior heterogeneity $[18,19,20]$ or stochastic components in the interaction. Generally speaking, macroscopic and mesoscopic models consider pedestrian flow as a continuum and deal with large modeling scales, while microscopic models operate at local scales. Yet a systematic classification of model features according to the model form is difficult. We refer to [21, 22, 23, 24, 25, 26, 27] for overviews of modeling approaches for pedestrian dynamics.

We aim in this article to tackle pedestrians' interactions as well as their granular aspects (e.g. pedestrian shape and collision-free property) and to describe their dynamics locally in simple geometries such as corridors and bottlenecks. Objectives are mainly addressed on the microscopic modeling scale.

Microscopic models are largely used in traffic engineering to simulate pedestrian dynamics. They describe pedestrians individually and can naturally take into account the heterogeneity and stochasticity of the pedestrians' behavior. Most of the models can reproduce fairly well several collective phenomena in pedestrian dynamics [26, 28, 29]. After more than 50 years of development, many kinds of microscopic models exist in the literature. We can distinguish between cellular automate models [30, 31, 32, 33, 34] (0th order models), velocity models [35, 36, 37, 38] (1st order models) and force-based models [39, 40, 41, 42] (2nd order models). While the former models are discrete in space and computationally fast, the later models are continuous in space and hence are easier to be used in complex geometries. Whether continuous models are computationally expensive depends not only on the order of the model but also on its definition. However, generally speaking, first-order models are less expensive since their numerical solution involves only one integration step, while two integration steps are required for second-order models. Furthermore, for numerical reasons fine discretization generally requires small integration time steps. In any case, microscopic models remain however generally much more computationally expensive than continuum pedestrian models. 
In this paper, we focus on the extension of the collision-free velocity model introduced in [36]. The collision-free velocity model (for short CVM) is a velocity-model, composed of a speed and a direction sub-models. Unlike most force-based models, CVM, being a first-order model, is by definition collision-free.

We generalize the CVM by considering the influence of walls and integrating two extensions. First, we change the shape of agents from circle to dynamical ellipse. In the original model, circles are used to express the projection of the pedestrian's body on the two-dimensional plane. However, many references and researches indicate that a dynamical ellipse can represent pedestrian's shape more accurately since the space a pedestrian occupied is influenced by the length of the legs during the motion and the lateral swaying of the body [40]. Therefore, we generalize CVM by extending the distance calculation to velocity-based ellipse and compare the simulation results with the original model (circles). After introducing the first extension, an unnatural "shaking" was observed during the simulation, which is caused by the zero-order direction sub-model. We propose a new first-order direction sub-model, designed to stabilize the direction changes of pedestrians in the simulation.

For the sake of completeness, we briefly introduce the original CVM in section 2. The generalization of the model from circle-based to an ellipse-based definition and the new direction sub-model are presented in section 3. In section 4, the comparison between the simulation results of a circle and a velocity-based ellipse is given and the performances of the new direction sub-model are compared to the original CVM. Finally, we give a summary of the extensions and discuss limitations of the model as well as future research directions in the concluding section 5 .

\section{Collision-free velocity model}

In the original model, the moving direction and speed of each pedestrian are updated at each time step. Moving direction of a pedestrian is obtained by superposing the influence of the surrounding pedestrians and the desired moving direction. The value of the speed depends on the minimum spacing in the moving direction. In figure 1 (borrowed from [36]), pedestrians are modeled as circles with constant diameter $\ell . X_{i}, X_{j}$ and $X_{k}$ are positions of pedestrians $i, j$ and $k$. The original CVM is described as

$$
\dot{X}_{i}\left(X_{i}, X_{j}, \ldots\right)=V_{i}\left(X_{i}, X_{j}, \ldots\right) \cdot \vec{e}_{i}\left(X_{i}, X_{j}, \ldots\right),
$$

where $V_{i}$ is the speed of pedestrian $i$ and $\vec{e}_{i}$ is the moving direction.

Moving direction $\vec{e}_{i}$ is obtained from the direction sub-model

$$
\vec{e}_{i}\left(X_{i}, X_{j}, \ldots\right)=u_{1} \cdot\left(\vec{e}_{i}^{0}+\sum_{j \in N_{i}} R\left(s_{i, j}\right) \cdot \vec{e}_{i, j}\right),
$$

where $u_{1}$ is a normalization constant such that $\left\|\vec{e}_{i}\right\|=1, \vec{e}_{i}{ }^{0}$ is the desired direction towards a certain goal, $N_{i}$ is the set containing all the neighbours of the pedestrian $i, \vec{e}_{i, j}$ is the 


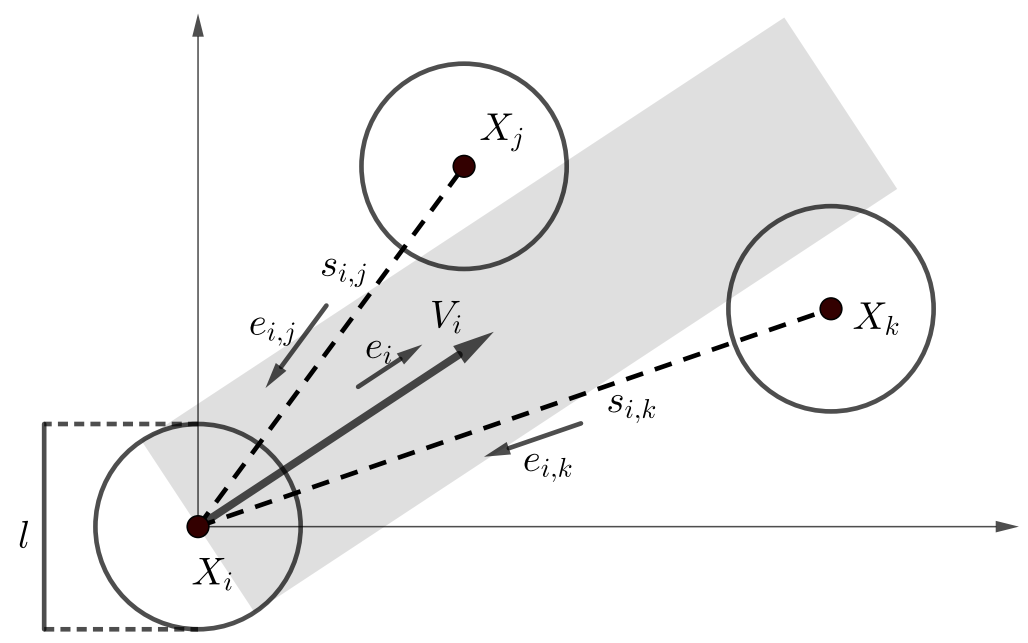

Figure 1: Notations used in the collision-free velocity model. $\ell$ is the size of agents, $X_{i}, X_{j}$ and $X_{k}$ are positions of pedestrians, $\vec{e}_{i}$ is the moving direction of pedestrian $i$ and $V_{i}$ is the moving speed, $s_{i, j}$ and $s_{i, k}$ are distances between the centers of pedestrians, $\vec{e}_{i, j}$ and $\vec{e}_{i, k}$ and the unit vector from $X_{j}$ and $X_{k}$ to $X_{i}$.

unit vector from the center of the pedestrian $j$ towards the center of the pedestrian $i$. The function

$$
R\left(s_{i, j}\right)=k \cdot \exp \left(\frac{\ell-s_{i, j}}{D}\right)
$$

is used to describe the influence that neighbours act on the moving direction of pedestrian $i$. The strength coefficient $k>0$ and the distance coefficient $D>0$ calibrate the function accordingly. As mentioned before, $\ell$ is the diameter of the circle used to represent the pedestrians and $s_{i, j}$ is the distance between the centers of pedestrian $i$ and $j$.

After obtaining the moving direction $\vec{e}_{i}$, the speed model

$$
V_{i}\left(s_{i, j}\right)=\min \left\{V_{i}^{0}, \max \left\{0, \frac{s_{i}-\ell}{T}\right\}\right\},
$$

is used to determine the scale of velocity $V_{i}$ in the direction $\vec{e}_{i}$. In Eq. (4), $V_{i}^{0}$ is the desired speed of pedestrian $i$, which depends on various environmental factors such as the existence of stairs or smoke produced by a fire.

$$
s_{i}=\min _{j \in J_{i}} s_{i, j},
$$

is the distance between the center of pedestrian $i$ and the center of the closet pedestrian in front of pedestrian $i$, when pedestrian $i$ moving in the direction $\vec{e}_{i}$. The definition of set $J_{i}$ in Eq. (5) is

$$
J_{i}=\left\{j, \vec{e}_{i} \cdot \vec{e}_{i, j} \leq 0 \text { and }\left|\vec{e}_{i}^{\perp} \cdot \vec{e}_{i, j}\right| \leq \frac{\ell}{s_{i, j}}\right\}
$$

where $\vec{e}_{i}^{\perp} \cdot \vec{e}_{i}=0 . J_{i}$ is the set of all pedestrians overlapping with the grey area in figure 1 . The only coefficient in the speed model is $T>0$ which is used to adjust the gap between pedestrians. 
The above-mentioned definition of the CVM describes specifically interactions among pedestrians. However, the influence of walls and obstacles has been left from the definition of the model. In this work, we close this gap by only considering straight walls. If the shape of the wall in the simulation is irregular, then we will approximate it to a few straight walls. In figure $2, X_{i}, \vec{e}_{i}$ and $V_{i}$ have the same definitions as in figure 1. Besides, there are two walls in the figure, wall $v$ and $w . C_{v}$ and $C_{w}$ are the closest points in wall $v$ and $w$ to the center of pedestrian $i$ respectively. $\vec{e}_{i, v}$ and $\vec{e}_{i, w}$ are the unit vectors from $C_{v}$ and $C_{w}$ to $X_{i}$. $s_{i, v}$ and $s_{i, w}$ are the distances from $C_{v}$ and $C_{w}$ to $X_{i}$. The angle between $\vec{e}_{i}$ and $-\vec{e}_{i, v}$ is $\alpha_{v}$ and the angle between $\vec{e}_{i}$ and $-\vec{e}_{i, w}$ is $\alpha_{w}$.

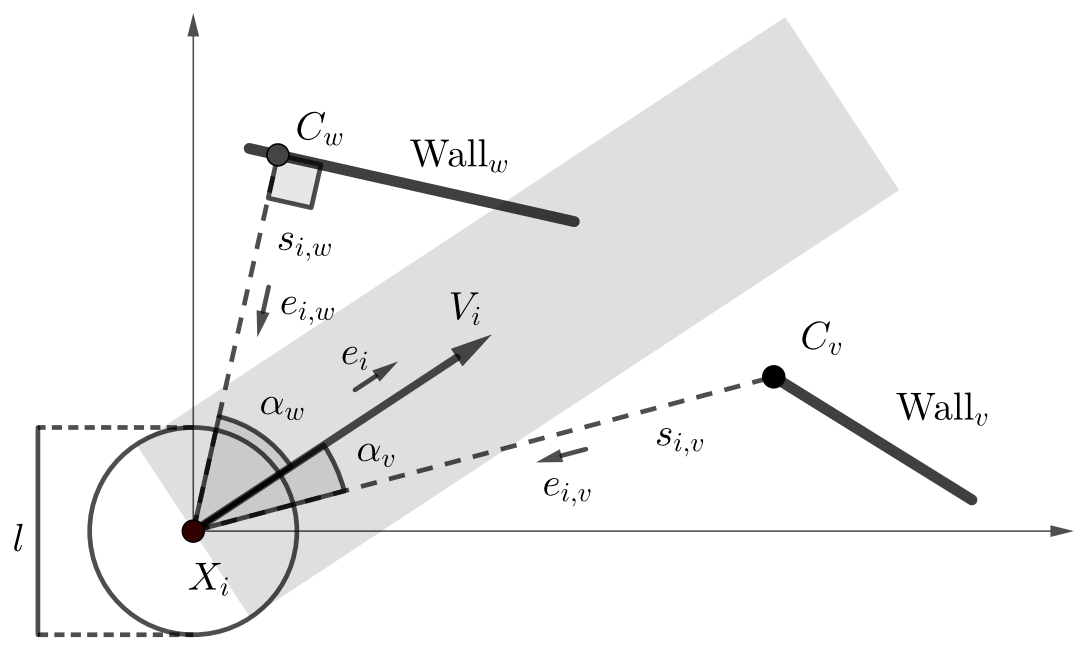

Figure 2: Notations used in the collision-free velocity model when calculating the influence of walls. $\ell, X_{i}$, $\vec{e}_{i}, V_{i}$ are the size, position, moving direction and moving speed of pedestrian $i, C_{v}$ and $C_{w}$ are the closest points in wall $v$ and $w$ to $X_{i}, \vec{e}_{i, v}$ and $\vec{e}_{i, w}$ are the unit vectors from $C_{v}$ and $C_{w}$ to $X_{i}, s_{i, v}$ and $s_{i, w}$ are the distances from $C_{v}$ and $C_{w}$ to $X_{i}, \alpha_{v}$ is the angle between $\vec{e}_{i}$ and $-\vec{e}_{i, v}, \alpha_{w}$ is the angle between $\vec{e}_{i}$ and $-\vec{e}_{i, w}$.

After introducing the influence of walls, the direction model becomes

$$
\vec{e}_{i}=u_{2} \cdot\left(\vec{e}_{i}^{0}+\sum_{j \in N_{i}} R\left(s_{i, j}\right) \cdot \vec{e}_{i, j}+\sum_{v \in W_{i}} R_{w}\left(s_{i, v}\right) \cdot \vec{e}_{i, v}\right),
$$

where $u_{2}$ is a normalization constant such that $\left\|\vec{e}_{i}\right\|=1, W_{i}$ is the set of walls nearby pedestrian $i$, and

$$
R_{w}\left(s_{i, v}\right)=k_{w} \cdot \exp \left(\frac{\frac{\ell}{2}-s_{i, v}}{D_{w}}\right)
$$

where $k_{w}>0$ and $D_{w}>0$ are used to calibrate the function accordingly.

To avoid overlaps of pedestrians with walls, walls should not only influence pedestrian's moving direction but also their speed. The expanded speed model is

$$
V_{i}=\min \left\{V_{i}^{0}, \max \left\{0, \frac{s_{i}-\ell}{T}\right\}, \max \left\{0, \frac{s w_{i}}{T}\right\}\right\}
$$


where the definitions of $s_{i}, \ell, T$ are same as in Eq. (4) and

$$
s w_{i}=\min _{v \in J W_{i}} \frac{s_{i, v}-\frac{\ell}{2}}{\cos \alpha_{v}}
$$

where $J W_{i}$ is the set containing all the walls in the moving direction of pedestrian $i$ (grey area in figure 2).

\section{Generalization of the collision-free velocity model}

In this section we introduce extensions of the CVM. We also show how every extension influences the resulting dynamics and eventually enhances the simulation results.

\subsection{From circle to ellipse}

We generalize the collision-free velocity model by extending the distance calculations to velocity-based ellipses. The plane view of the pedestrian $i$ 's body is represented by an ellipse [43]. The major semi-axis $a_{i}$ and minor semi-axis $b_{i}$ of the ellipse represent the space requirement in the direction of motion and along the shoulder axis respectively.

In [40] the semi-axis along the walking direction is defined as

$$
a_{i}=a_{\min }+\tau_{a} V_{i}
$$

where $V_{i}$ is the speed of pedestrian $i$, while $a_{\min }>0$ and $\tau_{a}>0$ are two parameters.

The idea that the semi-axis of the ellipse along the walking direction vary with speed is derived from the fact that the spacing a pedestrian needed in her moving direction has a positive correlation with her speed [44]. This, in turn, is also the role of parameter $T$ which is defined in the speed sub-model to adjust the gap between agents. We conclude that in our model $T$ and $\tau_{a}$ model the same behavior of pedestrians even if their physical interpretations are different.

This becomes apparent after performing a basic stability analysis of the model. Assuming an one-dimensional system in steady-state, we can derive from the speed sub-model in Eq. (4) the following relation

$$
V_{\text {steady }}=\frac{1 / \rho_{\text {steady }}-2 \cdot a_{\text {min }}}{\tilde{T}},
$$

where $V_{\text {steady }}$ and $\rho_{\text {steady }}$ are the speed and the density of pedestrians flow in steady state, and $\tilde{T}=T+2 \tau_{a}$. Hence, the parameter $\tau_{a}$ and the parameter $T$ in speed sub-model have the same influence on the dynamics. To confirm our assumption we perform numerical simulations by varying these two parameters while maintaining a constant value of $\tilde{T}$. We can observe from figure 3 that although the values of $\tau_{a}$ are different in these simulations, the results obtained are almost identical when $\tilde{T}$ is constant. In the spirit of Occam's razor, we dispense with parameter $\tau_{a}$ and opt for a constant semi-axis $a_{i}$. 


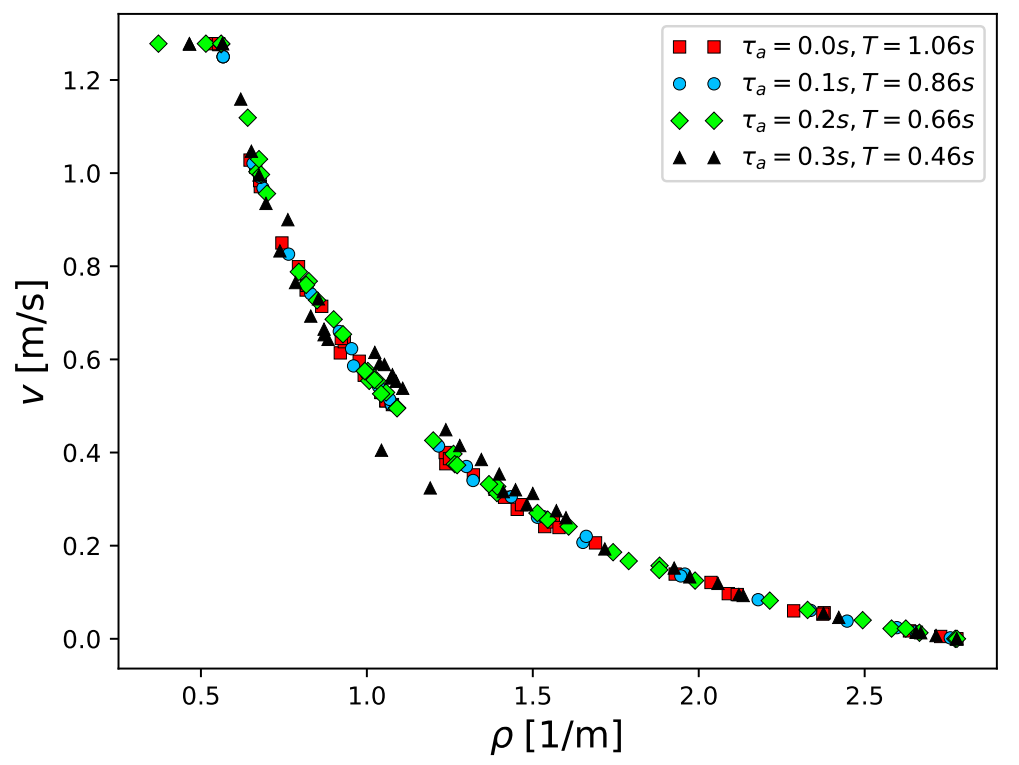

Figure 3: The fundamental diagram obtained in $1 \mathrm{D}$ simulations with $\tilde{T}=1.06$.

The other semi-axis along the shoulder axis $b_{i}$ is defined according to [40] as a linear function:

$$
b_{i}=b_{\max }-\left(b_{\max }-b_{\min }\right) \frac{V_{i}}{V_{i}^{0}},
$$

with $b_{\min }$ is the minimal semi-width when pedestrian $i$ reaches the desired speed $V_{i}^{0}$ and $b_{\max }$ is the maximum semi-width reached when pedestrian $i$ is not moving [40].

We found in simulations with the CVM that this linear relationship does not provide satisfactory results. Hence we introduce a new non-linear function inspired by the observation that pedestrians often reduce their occupied space in the vertical direction of motion by turning their body to walk faster and pass through narrow gaps that are smaller than the width of their shoulder.

We set

$$
b_{i}=b_{\min }+\frac{b_{\max }-b_{\min }}{1+e^{\beta \cdot\left(V_{i}-\gamma\right)}},
$$

which is a Sigmoid function, where the maximum semi-width $b_{\max }$ is equal to the half of a static pedestrian's width and $b_{\min }$ is equal to the half of a moving pedestrian's minimum width. Parameters $\beta$ and $\gamma$ are used to adjust the shape of the function as shown in figure 4 which shows the curves of the function for different parameter values.

After defining the semi-axes of the ellipse, we extend the distance calculations from circle to velocity-based ellipse (figure 5). The ellipses in the full line describe non-moving pedestrians, while the ellipses in the dashed line represent the pedestrians at the desired velocity. $d_{i, j}$ is the distance between ellipses used to represent pedestrian $i$ and $j$, which is defined as the distance between the borders of ellipses $i$ and $j$, along a line connecting their 


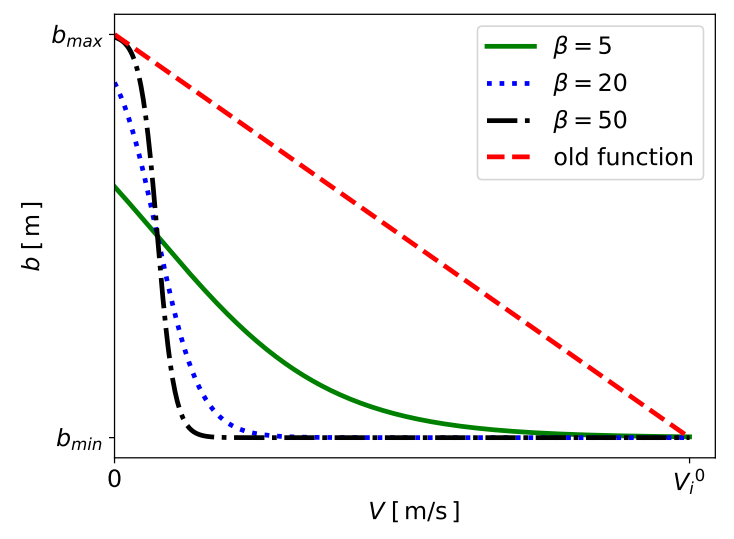

(a) $\gamma=0.1$

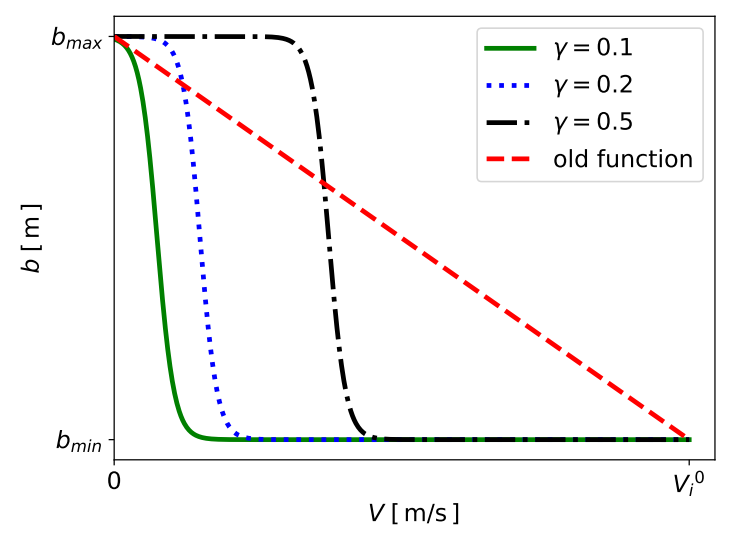

(b) $\beta=50$

Figure 4: The new function of $b$ with different $\beta$ and $\gamma$, the left figure shows the curves of the function with same $\gamma=0.1$ but different $\beta$ while the right figure shows the curves of the function with same $\beta=50$ but different $\gamma$.

centers. $d_{i, v}$ is the distance between the wall $v$ and pedestrian $i$, which is defined as the distance between the $C_{v}$ (the closest points in wall $v$ to the center of pedestrian $i$ ) and the border of ellipse used to present pedestrian $i$, along a line connecting the center of pedestrian $i$ and $C_{v}$.

In the new equation of motion, the influence of the agents' shape is added as follows: The moving direction $\vec{e}_{i}$ is calculated by Eq. (7), but the new definition of functions

$$
R\left(d_{i, j}\right)=k \cdot \exp \left(\frac{d_{i, j}}{D}\right), \quad R_{w}\left(d_{i, v}\right)=k_{w} \cdot \exp \left(\frac{d_{i, v}}{D_{w}}\right)
$$

are used. Then the speed $V_{i}$ is obtained by

$$
V_{i}=\min \left\{V_{i}^{0}, \max \left\{0, \frac{d_{i}}{T}\right\}, \max \left\{0, \frac{d w_{i}}{T}\right\}\right\}
$$

where

$$
d_{i}=\min _{j \in J_{i}} d_{i, j}, \quad d w_{i}=\min _{v \in J W_{i}} \frac{d_{i, v}}{\cos \alpha_{v}} .
$$

Here $J_{i}$ and $J W_{i}$ are the sets containing all pedestrians and walls in the direction of movement (i.e. the pedestrians and walls overlap with the grey area in figure 5). We set the width of the grey area to $2 b_{\min }$ in the case of a velocity-based ellipse. The comparisons between the models describing agents with different shapes are given in section 4 .

\subsection{New direction sub-model}

After generalizing the model to ellipses, some unrealistic phenomena during simulation become visible. First of all, backward movements occur very often, which is not realistic especially in evacuation scenarios. Second, an unnatural "shaking" appears during simulation, which is due to a strong fluctuation of the ellipse's orientation. 


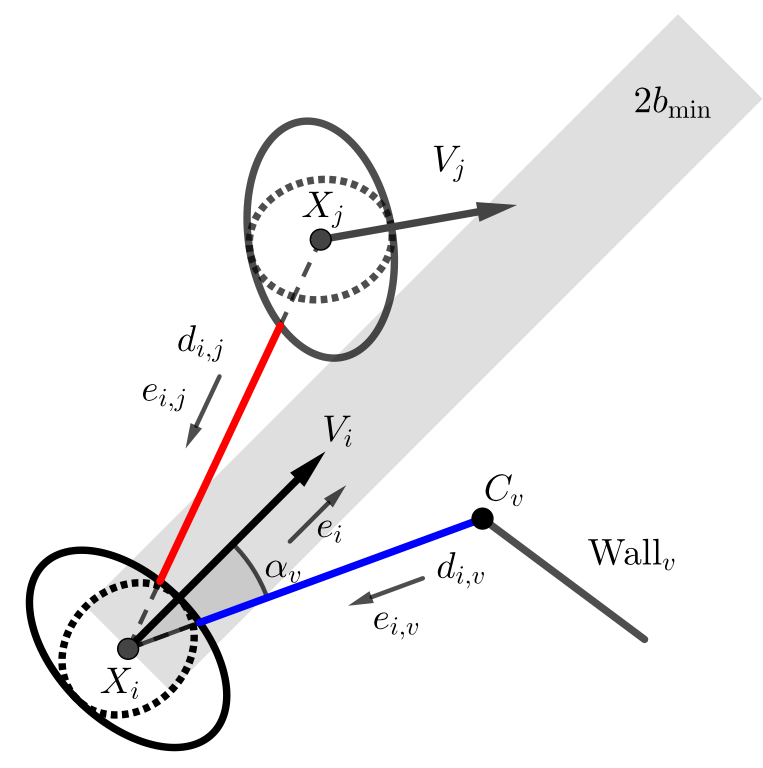

Figure 5: Notations used in the collision-free velocity model after extending the distance calculations between pedestrians from circle to velocity-based ellipse. $d_{i, j}$ is the length of red segment and $d_{i, v}$ is the length of blue segment. $X_{i}$ and $X_{j}$ are positions of pedestrians, $C_{v}$ is the closet point in wall $v$ to $X_{i}, V_{i}$ and $V_{j}$ are moving speeds of pedestrians, $\vec{e}_{i}$ is the moving direction of pedestrian $i, \vec{e}_{i, j}$ and $\vec{e}_{i, v}$ are the unit vectors from $X_{j}$ and $C_{v}$ to $X_{i}, \alpha_{v}$ is the angle between $\vec{e}_{i}$ and $-\vec{e}_{i, v}$.

In the original model, the moving direction of pedestrian $i$ is calculated by combining individual desired moving direction $\vec{e}_{i}^{0}$ and the neighbors' influence. Since the direction of neighbor's influence is from the center of pedestrians or closest point on the wall towards the center of the pedestrian $i$, the influence can be divided into two parts, one is the projection on $\vec{e}_{i}{ }^{0}$ and the other one is perpendicular to the projection part. The direction of the projection part is the reason for backward movements. Pedestrians hardly choose a moving direction whose projection on $\vec{e}_{i}^{0}$ is in the inverse direction of $\vec{e}_{i}^{0}$. And the cause of the "shaking" is that pedestrians turn to $\vec{e}_{i}$ directly after calculation in the original model (0th order model).

Therefore, our solution has two parts, the projection of neighbors' influence on $\vec{e}_{i}^{0}$ is always equal to zero, and introducing a smoothing process (e.g. a relaxation process) in the direction sub-model. Based on this idea, we propose a new direction sub-model as shown in figure 6 , where $\vec{e}_{i}{ }^{0}$ is the desired moving direction of the pedestrian $i$ and $\vec{e}_{i}$ is the actual moving direction, $\vec{e}_{i, j}^{N}$ and $\vec{e}_{i, v}^{N}$ are the new directions used to calculate the influence of the pedestrians $j$ and walls $v$ act on pedestrian $i$ respectively.

The new direction sub-model uses two steps to calculate the moving direction of a pedestrian. First, we use

$$
\vec{E}_{i}=u_{3} \cdot\left(\vec{e}_{i}^{0}+\sum_{j \in N_{i}} R\left(d_{i, j}\right) \cdot \vec{e}_{i, j}^{N}+\sum_{v \in W_{i}} R_{w}\left(d_{i, v}\right) \cdot \vec{e}_{i, v}^{N}\right),
$$

to calculate the optimal moving direction of the pedestrian $i, u_{3}$ is a normalization constant such that $\left\|\vec{E}_{i}\right\|=1$. The repulsive function $R\left(d_{i, j}\right)$ and $R_{w}\left(d_{i, v}\right)$ are given in Eq. (15) and 


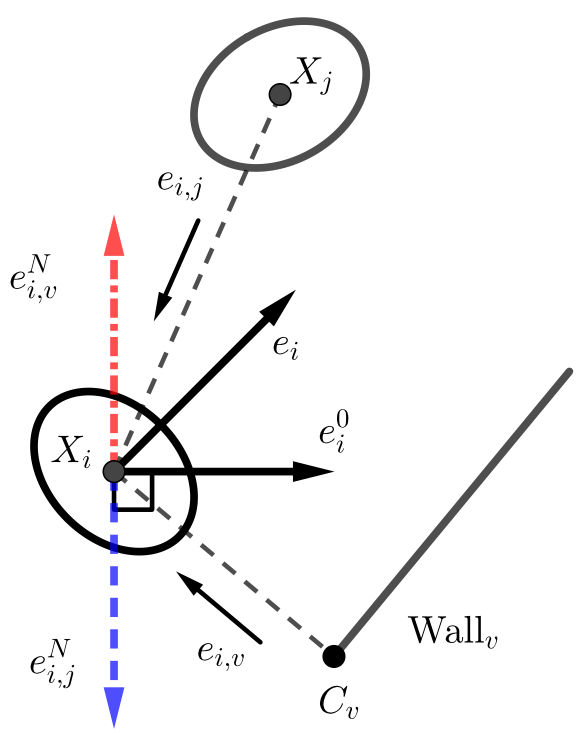

Figure 6: Notations used in the new direction sub-model. the direction of influence from pedestrian $j$ to pedestrian $i$ is vector represented by red chain line and the direction of influence from wall $v$ to pedestrian $i$ is vector represented by the blue dashed line. $X_{i}$ and $X_{j}$ are positions of the pedestrians, $C_{v}$ is the closet point in wall $v$ to $X_{i}, \vec{e}_{i}^{0}$ and $\vec{e}_{i}$ are the desired moving direction and the actual moving direction of the pedestrian $i, \vec{e}_{i, j}$ and $\vec{e}_{i, v}$ are the unit vectors from $X_{j}$ and $C_{v}$ to $X_{i}, \vec{e}_{i, j}^{N}$ and $\vec{e}_{i, v}^{N}$ are the directions used to calculate the influence of the pedestrians $j$ and walls $v$ act on pedestrian $i$ respectively.

the definition of $\vec{e}_{i, j}^{N}$ and $\vec{e}_{i, v}^{N}$ are

$$
\vec{e}_{i, j}^{N}=\left\{\begin{array}{ll}
\vec{e}_{i}{ }^{0 \perp} & \text { if } C_{j}>0, \\
\vec{e}_{i}{ }^{0 \perp} \text { or }-\vec{e}_{i}{ }^{0 \perp} & \text { if } C_{j}=0, \\
-\vec{e}_{i}{ }^{0 \perp} & \text { if } C_{j}<0 .
\end{array} \quad \vec{e}_{i, v}^{N}= \begin{cases}\vec{e}_{i}^{0 \perp} & \text { if } C_{v}>0, \\
\vec{e}_{i}^{0 \perp} \text { or }-\vec{e}_{i}^{0 \perp} & \text { if } C_{v}=0, \\
-\vec{e}_{i}^{0 \perp} & \text { if } C_{v}<0 .\end{cases}\right.
$$

where

$$
C_{j}=\vec{e}_{i, j} \cdot \vec{e}_{i}{ }^{0 \perp}, \quad C_{v}=\vec{e}_{i, v} \cdot \vec{e}_{i}^{0 \perp} .
$$

Here, $\vec{e}_{i}{ }^{0 \perp}$ is the vector obtained by rotating desired moving direction $\vec{e}_{i}{ }^{0}$ for $90^{\circ}$ counterclockwise. According to Eq. (19), influence from pedestrians and walls are decided not only by their position but also by the desired moving direction of the pedestrian $i$. If the centers of pedestrians or the closest points in walls to the center of the pedestrian $i$ are located in the left area to $\vec{e}_{i}^{0}$, the direction of influence is defined as right side perpendicular vector of $\vec{e}_{i}{ }^{0}$ and vice versa. It should be noticed that there might be an extremely rare case when $C_{j}$ or $C_{v}$ is equal to zero. In this case, the influence direction is decided by multiple factors, e.g. culture, gender. To simplify the model, the direction of influence is randomly chosen from $\vec{e}_{i}{ }^{0 \perp}$ and $-\vec{e}_{i}{ }^{0 \perp}$ in this case, corresponding to pedestrians avoiding front obstacles from the sides.

Then, we introduce a new relaxation time parameter $\tau$ in the direction sub-model, which 
is represented as

$$
\frac{d \vec{e}_{i}(t)}{d t}=\frac{\vec{E}_{i}(t)-\vec{e}_{i}(t)}{\tau}
$$

where $\vec{e}_{i}$ is the moving direction of the pedestrian $i$ and $\vec{E}_{i}$ is the optimal moving direction calculated by Eq. (18). In this step, we change the direction sub-module from zero-order to first-order, which does not change the global first-order property of the original CVM. By adjusting $\tau$, a pedestrian can turn to its moving direction smoothly.

Besides, we use a dynamical vision area in this paper, which is the hatching area in figure 7. Only the pedestrians and walls located in $\mathrm{Area}_{i}$, which is the dynamical vision area of the pedestrian $i$, influence the moving direction of the pedestrian $i$. The set contains all neighbors of the pedestrian $i$ in Area $_{i}$ is

$$
N_{i}^{\text {Area }}=\left\{j, \vec{e}_{i} \cdot \vec{e}_{i, j}<0 \text { or } \vec{e}_{i}^{0} \cdot \vec{e}_{i, j}<0\right\} .
$$

Here $\vec{e}_{i, j}$ is the vector from the center of neighbors towards the center of the pedestrian $i$. As for the walls, only when two vertices of a wall are both in Area $i$, this wall influences the moving direction of the pedestrian $i$. Vision area of the pedestrian $i$ is decided by his desired moving direction $\vec{e}_{i}{ }^{0}$ and his actual moving direction $\vec{e}_{i}$. This means a pedestrian choose the best moving direction according to the neighbors and walls located in the half area in front of his moving direction and the half area in front of his desired moving direction. The dynamical vision area is based on the idea that pedestrians will turn their heads to obtain the environmental information of the areas in front of their desired moving directions if their actual moving directions deviate from the desired moving directions. Using this dynamical vision area can eliminate some unrealistic block occurred between agents when using fixed vision area in the simulation.

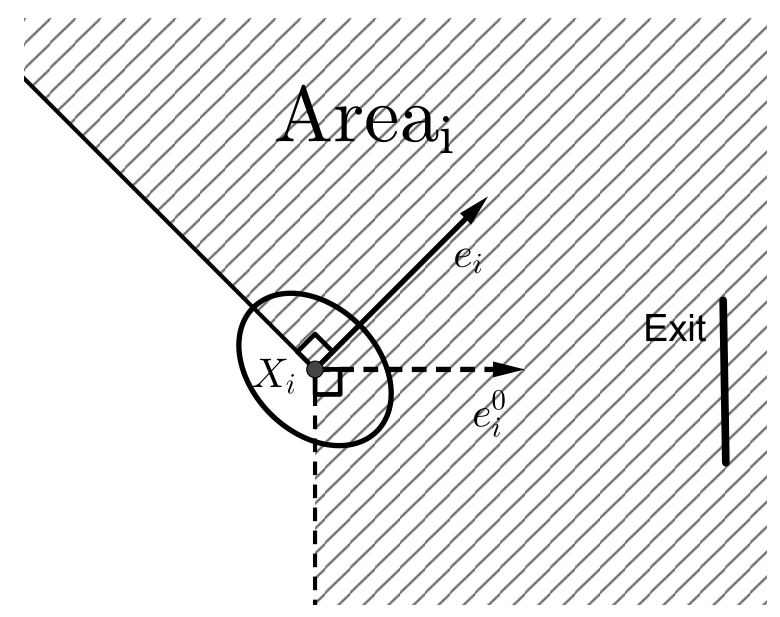

Figure 7: Dynamical vision area. $X_{i}$ is the position, Area is the hatching area, $\vec{e}_{i}{ }^{0}$ is the desired moving direction towards the exit, and $\vec{e}_{i}$ is the actual moving direction of pedestrian $i$.

These enhancements can almost eliminate the phenomena of backward movement and "shaking" in the simulation, as shown in figure 8. 


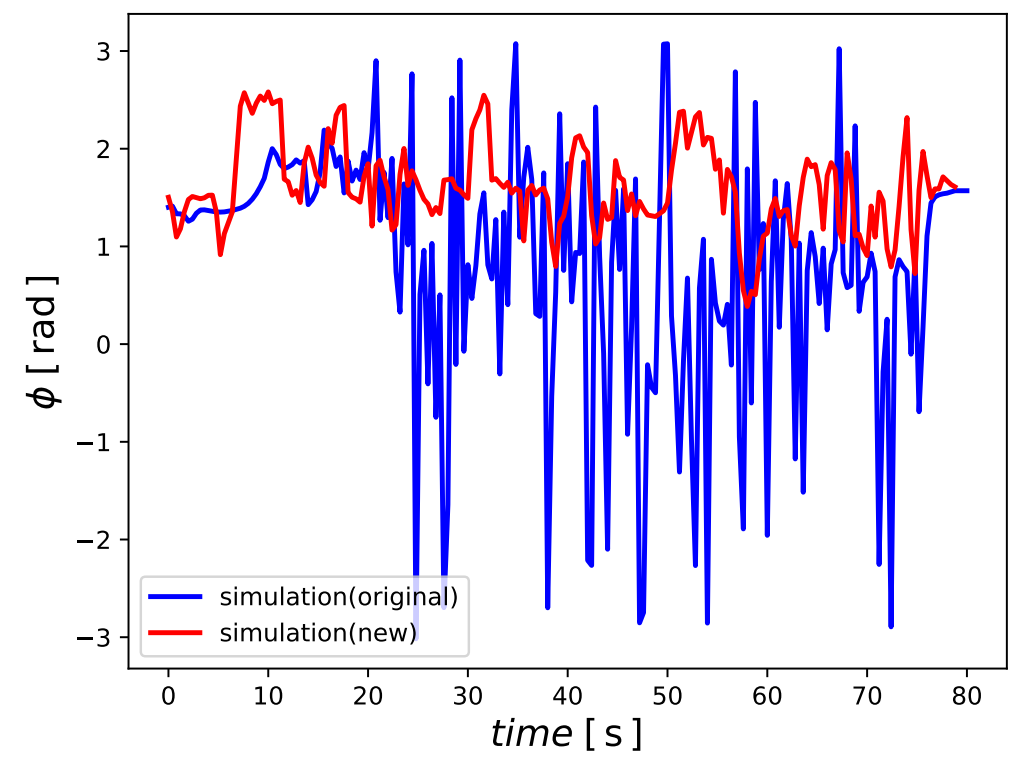

Figure 8: Time series of the angle between the moving direction of a pedestrian and the x-axis $(\phi)$.

$\phi$ is defined as the angle between the moving direction of a pedestrian and the $\mathrm{x}$-axis. As we can see in figure 8, the blue line (original model) shows a strong fluctuation of the angle over time compared with the red line (our extension).

In the next section, we further show a systematic comparison of both models.

\section{Simulation results}

In this section, the comparisons and analysis of models with different shapes and different direction sub-models are given. Preliminary simulation analysis has shown that the model can satisfy standards addressed in $[45,46]$ for basic movements of single pedestrians. We aim in this section to extend the validity of the model in regard to fundamental diagrams and collective behaviors in straight corridors and bottlenecks. The simulations in this section are executed with Euler scheme using a time step $\Delta t=0.05 \mathrm{~s}$. The update of the pedestrians is parallel in each step.

First, we perform simulations in a $26 \mathrm{~m}$ corridor with periodic boundary condition and measure the 1D fundamental diagram in a two meters long area located in the middle of the corridor. The shape of agents in these simulations, as well as the direction sub-model, are insignificant for the outcome of the simulation since pedestrians can not overtake others walking in front. Hence, we can focus on the validation of the speed sub-model and the relation between the speed and the required spacing in front.

The values of parameters are shown in table 1 . The desired speed of the pedestrians is $1.34 \mathrm{~m} / \mathrm{s}$. The shape of agents is circular with a constant radius $a$. The value of $a$ is $0.18 \mathrm{~m}$ 
and the value of $T$ is $1.06 \mathrm{~s}$, which are obtained from the linear relationship of required length and velocity [44].

Table 1: Parameters of CVM in one-dimensional scenario

\begin{tabular}{|c|c|c|c|c|c|c|c|}
\hline & $V^{0}(\mathrm{~m} / \mathrm{s})$ & $a(\mathrm{~m})$ & $T(\mathrm{~s})$ & $k$ & $D(\mathrm{~m})$ & $k_{w}$ & $D_{w}(\mathrm{~m})$ \\
\hline $1 \mathrm{D}$ & 1.34 & 0.18 & 1.06 & 3.0 & 0.1 & 6.0 & 0.05 \\
\hline
\end{tabular}

The simulation results in the $1 \mathrm{D}$ case are shown in figure 9. We realize that the obtained 1D fundamental diagram fit well with the experimental data.

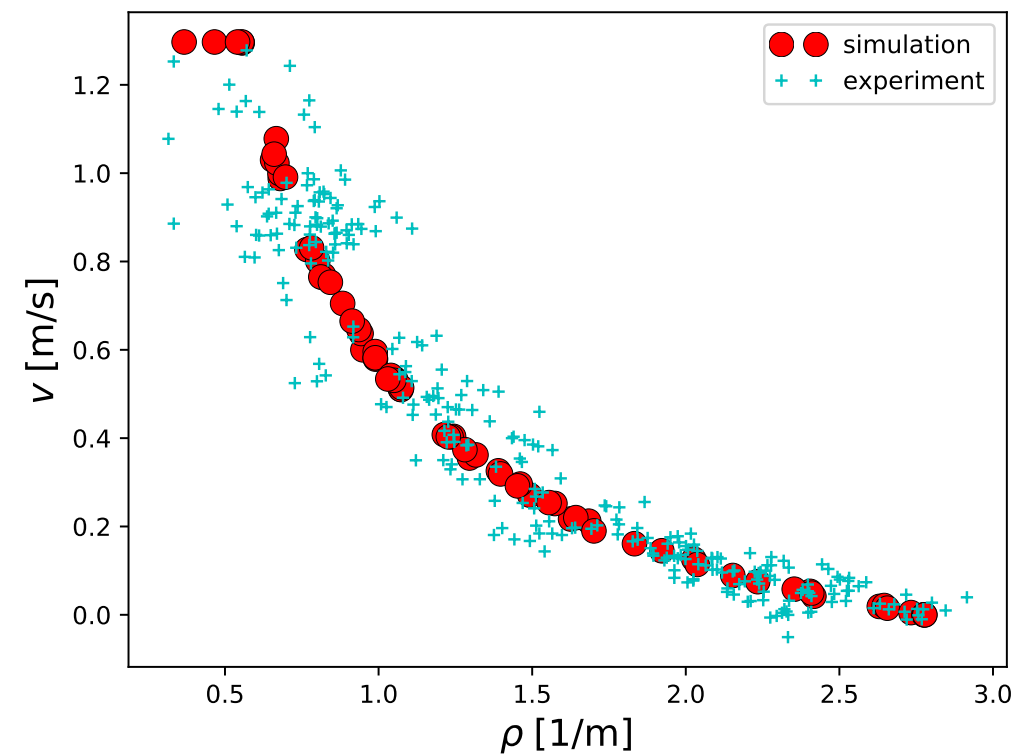

Figure 9: Velocity-density relation (fundamental diagram) in one-dimensional scenario, compared with experimental data [47].

In the second step, we investigate the effect of the agent's shape on the two-dimensional fundamental diagram. The simulation scenario is a $26 \times 1.8 \mathrm{~m}^{2}$ corridor with periodic boundary conditions. We measure the $2 \mathrm{D}$ fundamental diagram of models which describing agent with different shapes. We use three kinds of shapes here, circles with constant radius, ellipses with constant $a$ and variable $b$ as defined in Eq. (13) and ellipses with constant $a$ and variable $b$ as defined in Eq. (14).

The value of $V^{0}, a, T$ and parameters in direction sub-model are the same as in the one-dimensional case. Table 2 summarizes the value of other parameters.

The simulation results of the 2D case are shown in figure 10. From figure 10, we can get the result that the shape of agents in the model influence the fundamental diagram in the two-dimensional scenario, especially in the high-density area. The results obtained with constant circle and ellipse with variable $b$ defined as Eq. (13) both have deviation with 
Table 2: Parameters of CVM in two-dimensional scenario

\begin{tabular}{|c|c|c|c|c|c|}
\hline & $b_{\min }(\mathrm{m})$ & $b_{\max }(\mathrm{m})$ & $b$ function & $\beta$ & $\gamma$ \\
\hline constant circle & $\backslash$ & $\backslash$ & $\backslash$ & $\backslash$ & $\backslash$ \\
\hline original ellipse & 0.15 & 0.25 & $(13)$ & $\backslash$ & $\backslash$ \\
\hline new ellipse & 0.15 & 0.25 & $(14)$ & 50 & 0.1 \\
\hline
\end{tabular}

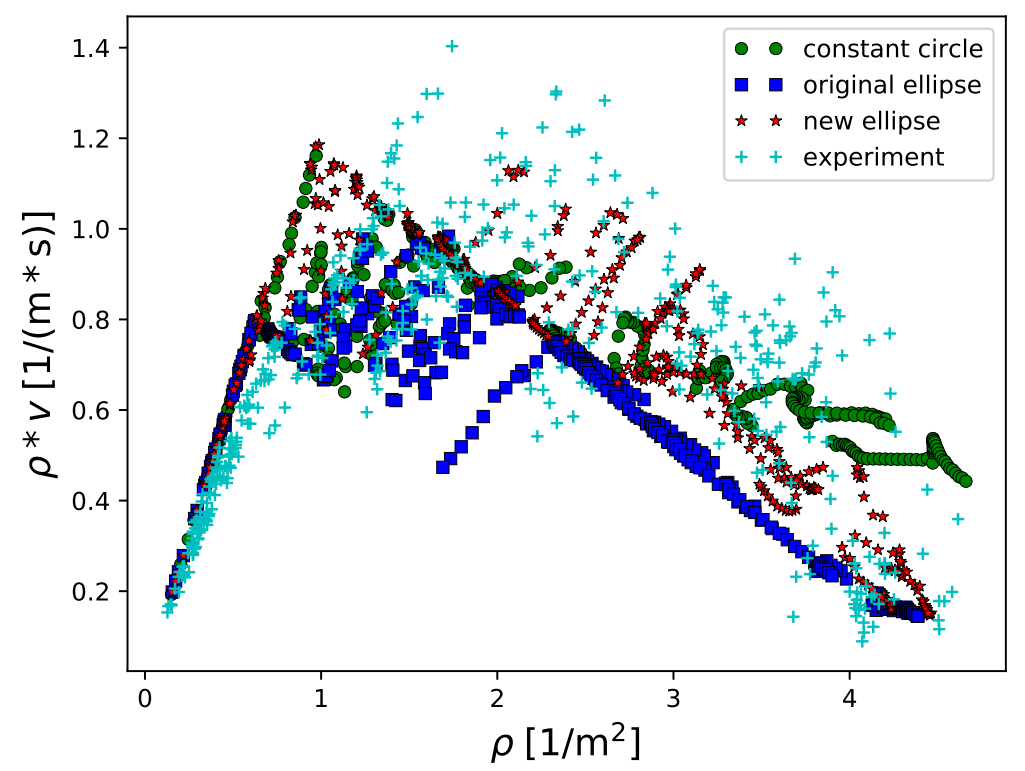

Figure 10: Relation between flow and density (fundamental diagram) in two-dimensional scenario, compared with experimental data obtained in the Hermes-project [48].

experimental data in the high-density area while using ellipse with variable $b$ defined as Eq. (14) can obtain 2D fundamental diagram which is closer to the experimental results. That means the new function for $b$ we proposed has a positive impact on the simulation result.

Then, we perform simulations in bottleneck scenarios [47]. We measure the relation between the flow in the middle of the bottleneck and the width of the bottleneck which is adjusted from $1.0 \mathrm{~m}$ to $2.5 \mathrm{~m}$ in our simulations. As we mentioned before, we can observe some unusual behavior during the simulation. Besides, we observe that the distribution of pedestrians in front of the bottleneck is different from the experiment. The new direction sub-model proposed in the previous section can eliminate these unusual phenomena.

In order to compare the simulation results of original and new direction sub-model fairly, we adjust the value of the parameter $T$ to make the flow-width relation obtained from the simulation results as close to the relation obtained from experimental data as possible. The shape of the pedestrian in original and new model are both the new dynamical ellipse we proposed in previous section, the value of $a, b_{\min }, b_{\max }, \beta$ and $\gamma$ are given in table 2 , the 
value of $k, D, k_{w}, D_{w}$ are provided in table 1 . The desired speeds of the pedestrians are Gaussian distributed with a mean of $1.34 \mathrm{~m} / \mathrm{s}$ and a standard deviation of $0.26 \mathrm{~m} / \mathrm{s}$ [49]. After validation, the value of $T$ in the original model is $0.5 \mathrm{~s}$ and in the new model is $0.45 \mathrm{~s}$. The value of new parameter $\tau$ introduced in new direction sub-model is $0.3 \mathrm{~s}$. The relations obtained are shown in figure 11 and compared with experimental data. In figure 11 we can find the relation obtained from simulation results of the original and new model both very close to the experimental data.

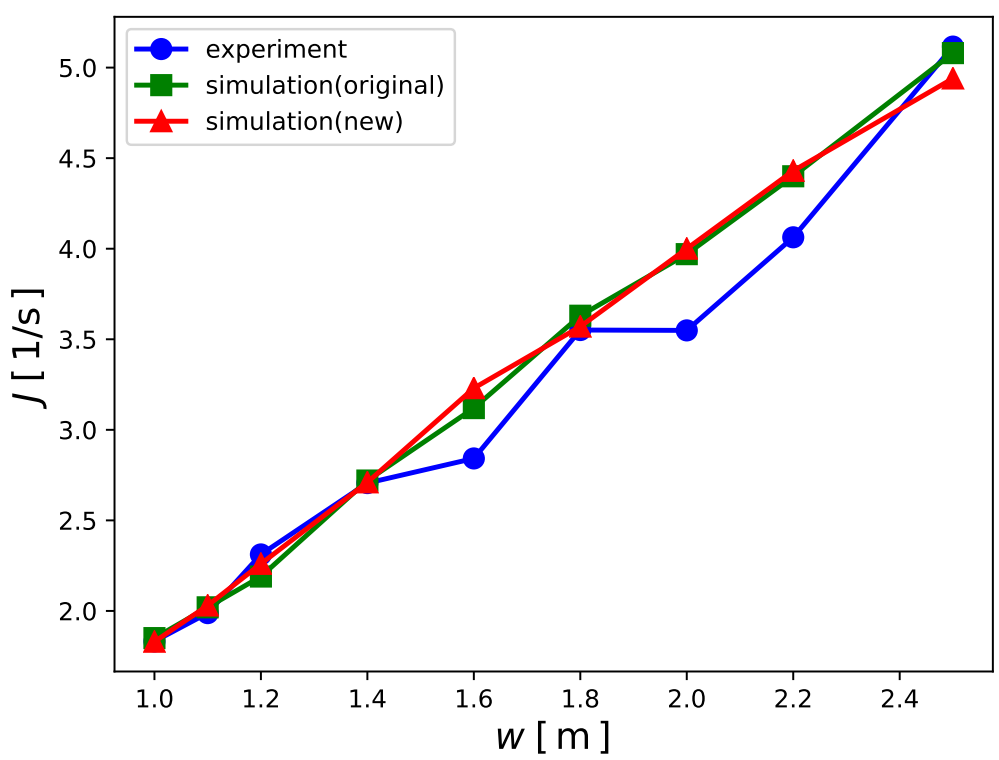

Figure 11: Relation between the flow in the middle of the bottleneck and the width of bottleneck, compared with experimental data[23].

Since the purpose of our extension is to eliminate backward movement and shaking phenomenon. We compare two indexes to prove that our extensions are useful. The first one is the backward movement proportion

$$
O=\frac{\sum_{i=1}^{N} \sum_{k=0}^{M_{i}} O_{i}(k \cdot \Delta t)}{\sum_{i=1}^{N} M_{i}},
$$

where $\Delta t$ is the time step size in the simulation, $M_{i} * \Delta t$ is the simulation duration of pedestrian $i, N$ is the number of pedestrians in the simulation and

$$
O_{i}(t)=\left\{\begin{array}{ll}
1, & \vec{e}_{i}(t) \cdot \vec{e}_{i}^{0}(t)<0 \\
0, & \text { else }
\end{array},\right.
$$


where $\vec{e}_{i}(t)$ is the moving direction of pedestrian $i$. This definition means that when the angle between the actual moving direction and the desired moving direction of a pedestrian is greater than 90 degrees, we regard it as a backward movement.

We calculate the proportion of backward movement from the simulation results of the original model and new model in bottleneck scenarios with different widths from $1.0 \mathrm{~m}$ to $2.5 \mathrm{~m}$. The results are shown and compared in figure 12 .

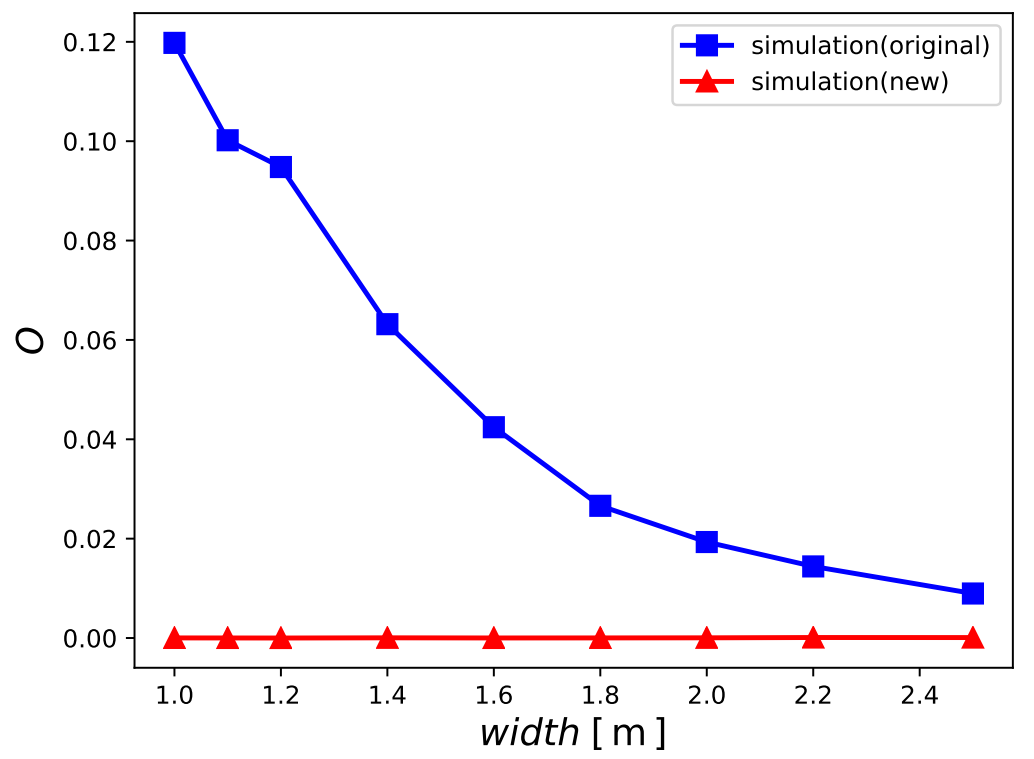

Figure 12: Proportion of backward movement in bottleneck scenarios with different widths from $1.0 \mathrm{~m}$ to $2.5 \mathrm{~m}$.

From figure 12, we can find that the proportion of backward movement significantly decreases in the new model compared to the original model. Therefore our extension eliminates the unrealistic backward movement.

The second index is the average angular variation in moving direction per pedestrian per frame, which is presented as

$$
S_{\text {average }}=\frac{\sum_{i=1}^{N} \sum_{k=1}^{M_{i}} S_{i}(k \cdot \Delta t)}{\sum_{i=1}^{N}\left(M_{i}-1\right)}
$$

with

$$
\left.S_{i}(t)=\mid \angle\left[\vec{e}_{i}(t), \vec{e}_{i} t-\Delta t\right)\right] \mid,
$$

where the definition of $\left.\angle\left[\vec{e}_{i}(t), \vec{e}_{i} t-\Delta t\right)\right]$ is the angle between $\vec{e}_{i}(t)$ and $\vec{e}_{i}(t-\Delta t)$. The definition of moving direction $\vec{e}_{i}(t)$ is the same as before. $S_{i}(t)$ is the absolute value of the 
angle between moving direction in the current time step and the previous one. We compare this index for the new model and the original model. The results are presented in figure 13 .

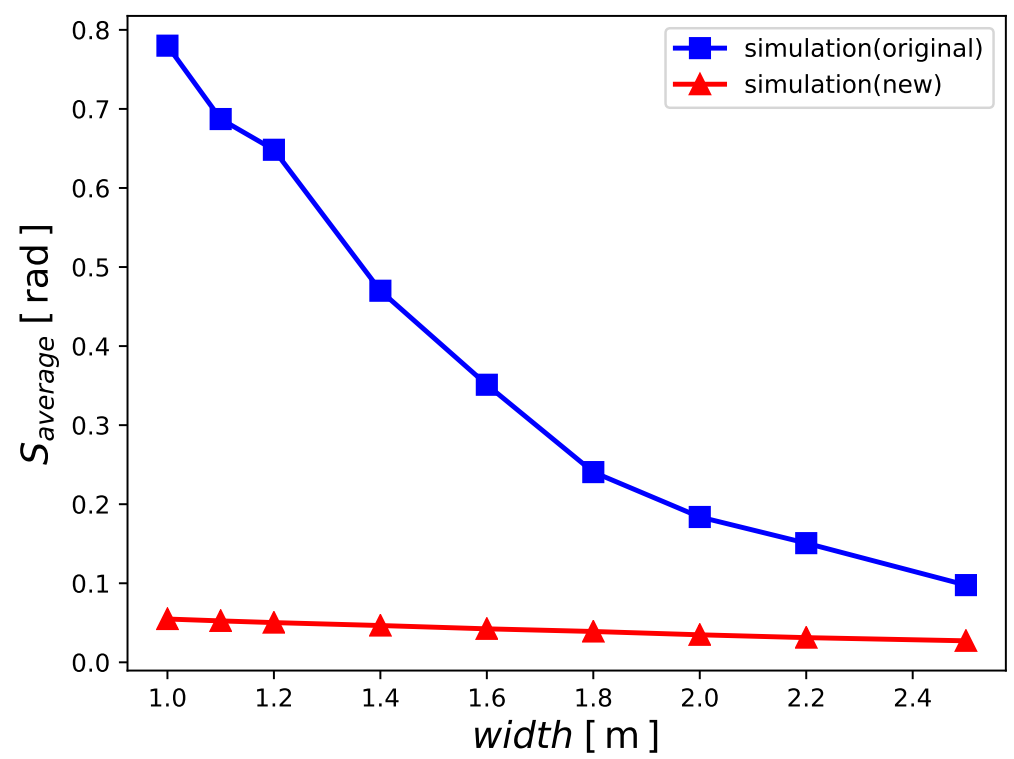

Figure 13: Average angular variation in moving direction per pedestrian per frame in bottleneck scenarios with different widths from $1.0 \mathrm{~m}$ to $2.5 \mathrm{~m}$.

It can be observed in figure 13 that in the new model the pedestrians change less their direction than the pedestrians in the original model, which is in line with the fact that pedestrians prefer to keep their direction instead of changing it. Compared within the original model, agents no longer shake frequently.

Finally, we compare the spatiotemporal profile of bottleneck flow when the width is $1.2 \mathrm{~m}$. In simulations, we initialise pedestrians in the same positions and at the same times as in the experiment, in order to eliminate the impact of pedestrians' initial distribution. The profiles obtained from the experiment, the original speed model as well as the new model are shown in figure 14. Although profiles obtained from new model are still somewhat different from the experimental results, a visible enhancement can be observed. The pedestrians do not deviate strongly from the exit as it can be observed with the original model.

\section{Conclusion}

In this paper we enhance and generalize the collision-free model [36] by introducing new components that lead to better dynamics. We firstly complete the collision-free velocity model by introducing the influence of walls. Then, we generalize the definition of the model in order to consider dynamical ellipse shapes of pedestrian's projection on the 2D space, instead of the originally used circular shapes. Hereby, we define the semi-axes of the ellipses such that the two-dimensional fundamental diagram is well reproduced with respect to 


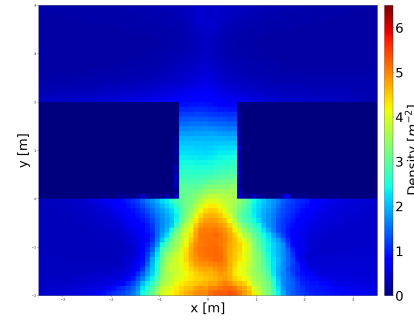

(a) density, experiment

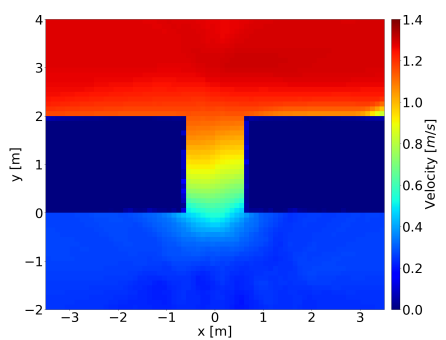

(d) velocity, experiment

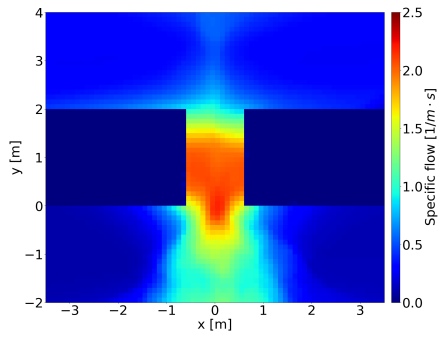

(g) Specific flow, experiment

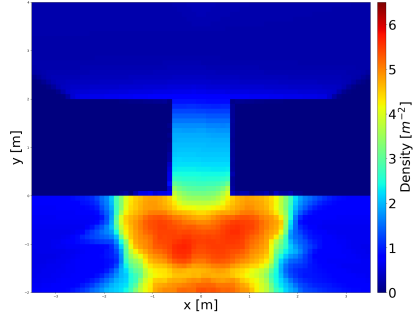

(b) density, original model

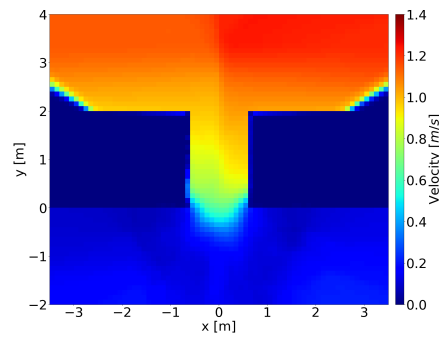

(e) velocity, original model

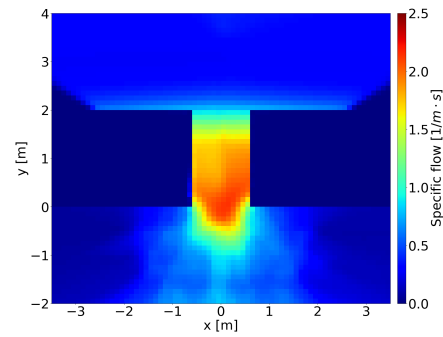

(h) Specific flow, original model

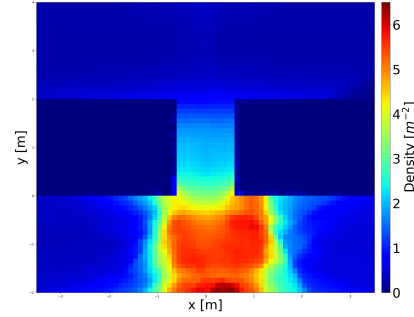

(c) density, new model

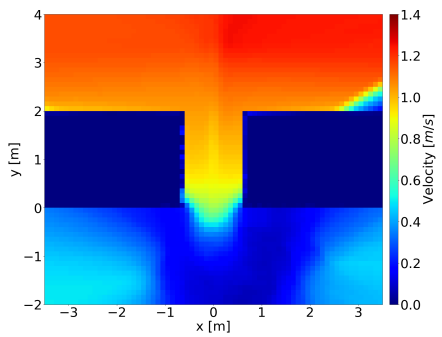

(f) velocity, new model

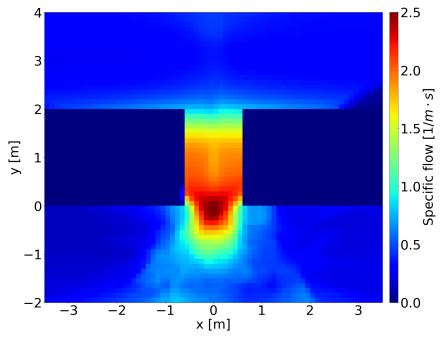

(i) Specific flow, new model

Figure 14: Spatiotemporal profile of bottleneck flow, the width of bottleneck is $1.2 \mathrm{~m}$. Pedestrians pass through the bottleneck from bottom to top. 
experimental data. After introducing a new direction sub-model, we show quantitatively that the unrealistic behavior of the agents during simulations with the original model could be mitigated. Simulation results show that the new direction sub-model can remove unrealistic backward movement and undesired shaking behaviors without compromising the benefits of the original model.

Our validation of the model was systematic, going from the fundamental diagram in narrow corridors (1D) through fundamental diagrams in wide corridors (2D) to the flowwidth relation in bottlenecks. Although the generalized model produces better results, there are still some problems that have not been solved yet. First of all, in bottleneck scenarios with small widths, a jamming arch may arise. Here, the collision-free nature of the model favors excessive blocking of agents in front of the exit. Further investigations are necessary to identify an appropriate mechanism for mitigating the effects of arching. Besides, more detailed validations will be done in future work.

\section{Acknowledgments}

Qiancheng $\mathrm{Xu}$ thanks the funding support from the China Scholarship Council (Grant NO.201706060186). Mohcine Chraibi thanks the support from the Visiting Professor International Project at the University of Science and Technology of China (2019A VR35).

\section{References}

[1] A. Seyfried, O. Passon, B. Steffen, M. Boltes, T. Rupprecht, W. Klingsch, New insights into pedestrian flow through bottlenecks, Transportation Science 43 (3) (2009) 395-406.

[2] D. C. Duives, W. Daamen, S. P. Hoogendoorn, State-of-the-art crowd motion simulation models, Transportation research part C: emerging technologies 37 (2013) 193-209.

[3] R. L. Hughes, A continuum theory for the flow of pedestrians, Transportation Research Part B: Methodological 36 (6) (2002) 507-535.

[4] J. A. Carrillo, S. Martin, M.-T. Wolfram, An improved version of the Hughes model for pedestrian flow, Mathematical Models and Methods in Applied Sciences 26 (04) (2016) 671-697.

[5] B. Piccoli, A. Tosin, Time-evolving measures and macroscopic modeling of pedestrian flow, Archive for Rational Mechanics and Analysis 199 (3) (2011) 707-738.

[6] S. P. Hoogendoorn, F. L. van Wageningen-Kessels, W. Daamen, D. C. Duives, Continuum modelling of pedestrian flows: From microscopic principles to self-organised macroscopic phenomena, Physica A: Statistical Mechanics and its Applications 416 (2014) $684-694$.

[7] A. Lachapelle, M.-T. Wolfram, On a mean field game approach modeling congestion and aversion in pedestrian crowds, Transportation Research Part B: Methodological 45 (10) (2011) 1572-1589.

[8] M. Burger, M. D. Francesco, P. A. Markowich, M.-T. Wolfram, Mean field games with nonlinear mobilities in pedestrian dynamics, Discrete \& Continuous Dynamical Systems - B 19 (2014) 1311.

[9] A. Aurell, B. Djehiche, Mean-field type modeling of nonlocal crowd aversion in pedestrian crowd dynamics, SIAM Journal on Control and Optimization 56 (1) (2018) 434-455.

[10] Y. Achdou, J.-M. Lasry, Contributions to Partial Differential Equations and Applications, Springer International Publishing, Cham, 2019, Ch. Mean Field Games for Modeling Crowd Motion, pp. 17-42.

[11] N. Bellomo, A. Bellouquid, On the modelling of vehicular traffic and crowds by kinetic theory of active particles, in: Mathematical modeling of collective behavior in socio-economic and life sciences, Springer, 2010, pp. 273-296.

[12] C. Dogbe, On the modelling of crowd dynamics by generalized kinetic models, Journal of Mathematical Analysis and Applications 387 (2) (2012) 512-532. 
[13] N. Bellomo, D. Clarke, L. Gibelli, P. Townsend, B. Vreugdenhil, Human behaviours in evacuation crowd dynamics: from modelling to big data toward crisis management, Physics of life reviews 18 (2016) 1-21.

[14] N. Bellomo, L. Gibelli, N. Outada, On the interplay between behavioral dynamics and social interactions in human crowds, Kinetic \& Related Models 12 (2019) 397.

[15] A. Elmoussaoui, P. Argoul, M. E. Rhabi, A. Hakim, Discrete kinetic theory for 2d modeling of a moving crowd: Application to the evacuation of a non-connected bounded domain, Computers \& Mathematics with Applications 75 (4) (2018) 1159-1180.

[16] A. Treuille, S. Cooper, Z. Popović, Continuum crowds, ACM Trans. Graph. 25 (3) (2006) 1160-1168.

[17] K. Rahman, N. A. Ghani, A. A. Kamil, A. Mustafa, M. K. Chowdhury, Modelling pedestrian travel time and the design of facilities: A queuing approach, PLoS ONE 8(5) (2013) e63503.

[18] A. Tordeux, G. Lämmel, F. S. Hänseler, B. Steffen, A mesoscopic model for large-scale simulation of pedestrian dynamics, Transportation Research Part C: Emerging Technologies 93 (2018) 128 - 147.

[19] G. Puppo, M. Semplice, A. Tosin, G. Visconti, Fundamental diagrams in traffic flow: The case of heterogeneous kinetic models, Communications in mathematical sciences 14 (2016) 643-669.

[20] G. Visconti, M. Herty, G. Puppo, A. Tosin, Multivalued fundamental diagrams of traffic flow in the kinetic Fokker-Planck limit, Multiscale Modeling \& Simulation 15 (2017) 1267-1293.

[21] N. Bellomo, A. Bellouquid, D. Knopoff, From the microscale to collective crowd dynamics, Multiscale Modeling \& Simulation 11 (3) (2013) 943-963.

[22] E. Cristiani, B. Piccoli, A. Tosin, Multiscale modeling of pedestrian dynamics, Vol. 12, Springer, 2014.

[23] N. Bellomo, L. Gibelli, Toward a mathematical theory of behavioral-social dynamics for pedestrian crowds, Mathematical Models and Methods in Applied Sciences 25 (13) (2015) 2417-2437.

[24] N. Bellomo, C. Dogbe, On the modeling of traffic and crowds: A survey of models, speculations, and perspectives, SIAM review 53 (3) (2011) 409-463.

[25] N. Bellomo, A. Bellouquid, On multiscale models of pedestrian crowds from mesoscopic to macroscopic, Commun. Math. Sci 13 (7) (2015) 1649-1664.

[26] M. Chraibi, A. Tordeux, A. Schadschneider, A. Seyfried, Modelling of pedestrian and evacuation dynamics, Encyclopedia of Complexity and Systems Science (2018) 1-22.

[27] L. Gibelli, N. Bellomo, Crowd Dynamics - Theory, Models, and Safety Problems, Vol. 1, Springer, 2019.

[28] D. Helbing, I. J. Farkas, P. Molnar, T. Vicsek, Simulation of pedestrian crowds in normal and evacuation situations, Pedestrian and evacuation dynamics 21 (2) (2002) 21-58.

[29] A. Schadschneider, A. Seyfried, Empirical results for pedestrian dynamics and their implications for modeling, Networks \& Heterogeneous Media 6 (2011) 545.

[30] V. J. Blue, J. L. Adler, Cellular automata microsimulation for modeling bi-directional pedestrian walkways, Transportation Research Part B: Methodological 35 (3) (2001) 293-312.

[31] C. Burstedde, K. Klauck, A. Schadschneider, J. Zittartz, Simulation of pedestrian dynamics using a two-dimensional cellular automaton, Physica A: Statistical Mechanics and its Applications 295 (3-4) (2001) 507-525.

[32] M. Fukui, Y. Ishibashi, Self-organized phase transitions in cellular automaton models for pedestrians, Journal of the physical society of Japan 68 (8) (1999) 2861-2863.

[33] A. Kirchner, A. Schadschneider, Simulation of evacuation processes using a bionics-inspired cellular automaton model for pedestrian dynamics, Physica A: statistical mechanics and its applications 312 (12) (2002) 260-276.

[34] M. Muramatsu, T. Irie, T. Nagatani, Jamming transition in pedestrian counter flow, Physica A: Statistical Mechanics and its Applications 267 (3-4) (1999) 487-498.

[35] A. Tordeux, A. Seyfried, Collision-free nonuniform dynamics within continuous optimal velocity models, Physical Review E 90 (4) (2014) 042812.

[36] A. Tordeux, M. Chraibi, A. Seyfried, Collision-free speed model for pedestrian dynamics, in: Traffic and Granular Flow'15, Springer, 2016, pp. 225-232.

[37] B. Maury, J. Venel, A discrete contact model for crowd motion, ESAIM: Mathematical Modelling and Numerical Analysis 45 (1) (2011) 145-168. 
[38] S. Paris, J. Pettré, S. Donikian, Pedestrian reactive navigation for crowd simulation: a predictive approach, in: Computer Graphics Forum, Vol. 26, Wiley Online Library, 2007, pp. 665-674.

[39] D. Helbing, P. Molnar, Social force model for pedestrian dynamics, Physical review E 51 (5) (1995) 4282.

[40] M. Chraibi, A. Seyfried, A. Schadschneider, Generalized centrifugal-force model for pedestrian dynamics, Physical Review E 82 (4) (2010) 046111.

[41] D. R. Parisi, M. Gilman, H. Moldovan, A modification of the social force model can reproduce experimental data of pedestrian flows in normal conditions, Physica A: Statistical Mechanics and its Applications 388 (17) (2009) 3600-3608.

[42] A. Johansson, D. Helbing, P. K. Shukla, Specification of the social force pedestrian model by evolutionary adjustment to video tracking data, Advances in complex systems 10 (supp02) (2007) 271-288.

[43] J. J. Fruin, Pedestrian planning and design, Tech. rep., New York: Elevator World (1971).

[44] A. Seyfried, B. Steffen, W. Klingsch, M. Boltes, The fundamental diagram of pedestrian movement revisited, Journal of Statistical Mechanics: Theory and Experiment 2005 (10) (2005) P10002.

[45] R. e.V., Guideline for microscopic evacuation analysis, URL https://rimeaweb.files.wordpress. com/2016/06/rimea_richtlinie_3-0-0_-_d-e.pdf acceded on 01.07.2019 (2016).

[46] E. Ronchi, E. D. Kuligowski, P. A. Reneke, R. D. Peacock, D. Nilsson, The process of verification and validation of building fire evacuation models, US Department of Commerce, National Institute of Standards and Technology, 2013.

[47] A. Seyfried, M. Boltes, J. Kähler, W. Klingsch, A. Portz, T. Rupprecht, A. Schadschneider, B. Steffen, A. Winkens, Enhanced empirical data for the fundamental diagram and the flow through bottlenecks, in: Pedestrian and Evacuation Dynamics 2008, Springer, 2010, pp. 145-156.

[48] S. Holl, A. Seyfried, Hermes-an evacuation assistant for mass events, Inside 7 (1) (2009) 60-61.

[49] S. Buchmüller, U. Weidmann, Parameters of pedestrians, pedestrian traffic and walking facilities, IVT Schriftenreihe 132 (2006). 